\title{
The association between coronary flow rate and impaired heart rate recovery in patients with metabolic syndrome: A preliminary report
}

\author{
Yusuf I. Alihanoglu ${ }^{1}$, I. Dogu Kilic ${ }^{1}$, Harun Evrengul ${ }^{1}$, Bekir S. Yildiz ${ }^{1}$, Ihsan Alur ${ }^{2}$, \\ Burcu Uludag ${ }^{1}$, Omur Kuru³, Ozgur Taskoylu ${ }^{4}$, Havane Asuman Kaftan ${ }^{1}$ \\ ${ }^{1}$ Department of Cardiology, Pamukkale University School of Medicine, Denizli, Turkey \\ ${ }^{2}$ Department of Cardiovascular Surgery, Pamukkale University School of Medicine, Denizli, Turkey \\ ${ }^{3}$ Department of Cardiology, Erpa Private Hospital, Denizli, Turkey \\ ${ }^{4}$ Department of Cardiology, Servergazi State Hospital, Denizli, Turkey
}

\begin{abstract}
Background: The aim of this study is to evaluate heart rate recovery (HRR) and association between coronary flow rate and HRR in patients with metabolic syndrome (MS) who had morphologically normal coronary angiogram.

Methods: Study population included 43 patients with MS and 37 control subjects without MS. All patients were selected from individuals who had recently undergone coronary angiography in our hospital and were diagnosed as having angiographically normal coronary arteries. Exercise stress test results obtained prior to coronary angiography were evaluated for calculating HRR and other parameters. In addition, coronary flow was objectively evaluated for each major coronary artery in each subject using TIMI frame count method.
\end{abstract}

Results: All HRR values calculated were detected significantly lower in MS group compared to controls (HRR first: $32 \pm 9$ vs. $37 \pm 10 ; p=0.01$, second: $46 \pm 11$ vs. $52 \pm 11 ; p=0.03$, third: $51 \pm 12$ vs. $59 \pm 12 ; p=0.00$, fourth: $54 \pm 13$ vs. $61 \pm 2 ; p=0.02$ ). TIMI frame counts for each major epicardial coronary artery and mean TIMI frame count were also found to be significantly higher in MS group compared to controls (left anterior descending artery: $51 \pm 24$ vs. $39 \pm 15 ; p=0.009$, left circumflex artery: $32 \pm 11$ vs. $24 \pm 7 ; p=0.001$, right coronary artery: $33 \pm 14$ vs. $24 \pm 10 ; p=0.003$, mean TIMI frame count: $38 \pm 15$ vs. $29 \pm 9$; $p=0.002)$. Additionally, significant negative correlations were also detected between HRR first minute and coronary TIMI frame count values in patients with MS. None of MS parameters did not affect HRR values, however mean TIMI frame count independently associated with HRR first minute $(p=0.04)$ in patients with MS.

Conclusions: Impaired coronary blood flow occurring in MS might be a clue of autonomic dysfunction in addition to previously known endothelial dysfunction. (Cardiol J 2014; 21, 3: 257-264)

Key words: metabolic syndrome, heart rate recovery, coronary flow rate, TIMI frame count

Address for correspondence: Yusuf I. Alihanoglu, MD, Department of Cardiology, Pamukkale University, Medical Faculty, Denizli, Turkey, tel: +905054506009, e-mail: aliizyu@mynet.com

Received: 25.05.2013 Accepted: 25.07.2013 


\section{Introduction}

Heart rate recovery (HRR) is defined as the difference of heart rate (HR) at peak exercise and at a specific time interval following the onset of recovery. HRR appears to be related to the balance of sympathetic and parasympathetic tonus, which is mediated partly by the alterations in venous return and stretch of atrial receptors. The rise in HR during exercise is considered to be due to the combination of parasympathetic withdrawal and sympathetic activation. Therefore, HRR is a reflection of vagal reactivation and impaired HRR is considered to represent decreased vagal tone $[1,2]$. Slow deceleration of HR after exercise was found to be an independent predictor of cardiovascular (CV) disease and all-cause mortality [3, 4]. Autonomic dysfunction, including impaired vagal reactivation, as well as sympathetic overactivity, is known to be associated with hyperinsulinemia or insulin resistance, which determines metabolic syndrome (MS) [5-7]. An association has been found between HRR and MS, as well as between HRR and each of the components of this syndrome [8,9].

In patients at high risk for coronary artery disease (CAD), such as patients with MS, endothelial dysfunction is observed even in morphologically intact vessels before the onset of clinically manifested vascular disease $[10,11]$. It was also demonstrated that coronary flow rate was decreased in patients with MS [12]. The Thrombolysis in Myocardial Infarction (TIMI) frame count is a simple clinical tool for assessing quantitative indexes of coronary blood flow. It has been suggested that a higher TIMI frame count may reflect endothelial dysfunction [13]. The effect of the autonomic nervous system on coronary blood flow and insulin resistance is well known, however, it has not been exactly established yet whether impaired coronary blood flow occurring in MS arises from endothelial dysfunction or autonomic dysfunction.

In this study, we aimed to evaluate HRR at various time intervals, and the association between coronary flow rate and HRR in patients with MS who had morphologically normal epicardial coronary arteries in coronary angiography.

\section{Method}

\section{Study population}

The study population included 43 patients with MS (group I: 22 men, 21 women; mean age $55 \pm 10$ years) and 37 control subjects without MS (group II: 22 men, 15 women; mean age
$51 \pm 8$ years). All patients were selected from the individuals who had recently undergone coronary angiography in our hospital with a suspicion of CAD and were diagnosed as having angiographically normal coronary arteries. The indications for coronary angiography in these patients were the presence of angina pectoris and/or abnormal noninvasive test results suggesting myocardial ischemia. Exercise stress test results of the patients obtained prior to the coronary angiography were evaluated for calculating HRR values and other parameters. Exclusion criteria included known CAD, history of myocardial infarction, left ventricular dysfunction, left ventricular hypertrophy, valvular heart disease, an implanted pacemaker, atrial fibrillation, hypo-hyperthyroidism, uncontrolled hypertension, and chronic respiratory disease. The use of any medications affecting blood lipid profile, glucose level, blood pressure (BP), and HR response to the exercise, such as digoxin, beta-blockers, or nondihydropyridine calcium channel blockers, were also accepted as exclusion criteria. In addition, only the patients whose exercise tests had been terminated due to reached target HR were enrolled in this study. This study was designed as a retrospective study; the study protocol was prepared in accordance with the Declaration of Helsinki and was approved by the local Ethic Committee.

\section{Medical examination}

Seated BP was measured 3 times after a 5-minute resting with the average of the last 2 measurements. Height and weight values were measured with the participants in light examination clothes without shoes. Waist circumference was measured using the average of 2 measurements between the iliac crest and the bottom of the ribcage while the subject was standing. Body mass index (BMI) was defined as weight in kilograms divided by the square of height in meter. Blood sampling was taken after at least $12 \mathrm{~h}$ fasting. The level of total cholesterol, high-density lipoprotein cholesterol (HDL-C), low-density lipoprotein cholesterol (LDL-C), triglycerides (TG), glucose, insulin, creatinine, and hemoglobin were measured by using routine laboratory techniques. Homeostasis model assessment index of insulin resistance (HOMA-R) was defined as fasting plasma insulin $[\mathrm{mu} / \mathrm{L}] \times$ fasting glucose $[\mathrm{mg} / \mathrm{dL}] / 405$ by using the Matthews's equation [14].

\section{Definition of metabolic syndrome}

We defined MS according to the definition of the Third Adult Treatment Panel (NCEP-ATP III) 
if 3 or more of the following components were met: (1) fasting blood glucose level $\geq 110 \mathrm{mg} / \mathrm{dL}$; (2) systolic $\mathrm{BP} \geq 130 \mathrm{~mm} \mathrm{Hg}$ or diastolic $\mathrm{BP} \geq 85 \mathrm{~mm} \mathrm{Hg}$; (3) TG level $\geq 150 \mathrm{mg} / \mathrm{dL}$; (4) HDL-C level $\leq 40 \mathrm{mg} / \mathrm{dL}$ in men or $\leq 50 \mathrm{mg} / \mathrm{dL}$ in women; and (5) waist circumference $>102 \mathrm{~cm}$ in men or $>88 \mathrm{~cm}$ in women.

\section{Protocol of the exercise stress test}

The patients underwent a standard symptom-limited, maximal-graded exercise treadmill test according to the standard Bruce protocol with a Quinton Treadmill system (Quinton. Inc., Bothell, WA, USA). Continuous, 12-lead electrocardiographic monitoring was performed throughout the testing. The Tango exercise BP monitoring (SunTech Medical, Morrisville, North Carolina) device was used to automatically measure the subjects' BP and HR before and at the second minute of each stage of the exercise. The participants exercised until limiting symptoms such as dyspnea, dizziness, fatigue, leg cramps, or if the HR achieved was more than $>95 \%$ of estimated maximal HR (220 - age). The patients continued to walk for $60 \mathrm{~s}$ at a speed of $1.5 \mathrm{mph}$ during the recovery period and they sat down with continued $\mathrm{BP}$ and $\mathrm{HR}$ monitoring. HRR values were calculated by subtracting the HR at the first, second, third, and fourth minutes of the recovery period from the HR reached at peak exercise. The exercise capacity was calculated as total metabolic equivalent units (METs) achieved at peak exercise. HR was measured at rest, during each minute of exercise, and at the first, second, and third minutes of the recovery period.

\section{Evaluation of the coronary blood flow}

Coronary flow was evaluated objectively by 2 independent observers using the TIMI frame count. TIMI frame count was determined for each major coronary artery in each subject according to the method first described by Gibson et al. [13]. The first frame was defined as the frame in which the full width of the proximal coronary artery lumen was filled with the concentrated dye, touching both borders of the lumen, and forward motion down the artery. The final frame was determined when the leading edge of the contrast column initially arrived at the distal landmark. The most distal branch nearest the apex of the left ventricle was used for the left anterior descending coronary artery (LAD), because the LAD is usually longer than the other major coronary arteries and the TIMI frame count for this vessel is often higher. Therefore, TIMI frame count was divided by 1.7 to obtain a corrected TIMI frame count for the LAD [13]. The distal landmark for the right coronary artery (RCA) was designated as the first branch of the posterolateral RCA after the origin of the posterior descending artery. The branch of the left circumflex artery (LCx) that encompassed the greatest total distance traveled by the contrast was used to define the distal landmark of the LCx. While the TIMI frame counts in the LAD and LCx were assessed in a right anterior oblique projection with caudal angulation, the RCA was assessed in a left anterior oblique projection with cranial angulation. The mean TIMI frame count for the individuals was calculated by adding the TIMI frame counts for the LAD, LCx, and RCA and then dividing the obtained value by 3 .

\section{Statistical analysis}

While continuous variables were expressed as mean \pm standard deviation, categorical variables were expressed as percentages. Comparisons of categorical and continuous variables between the two groups were performed using the $\chi^{2}$ test and unpaired t-test, respectively. The correlation between biochemical, anthropometric measurements, HRR values, and mean TIMI frame count was evaluated by the Pearson correlation test. Interobserver variability was determined by calculating the differences between the means of the 2 measurements made by 2 independent observers in the same patient. Measurement differences were converted into percentage differences, calculated as the difference between the measurements by the 2 observers divided by the average of the 2 measurements multiplied by 100 . Multivariate linear regression analysis was applied to evaluate the effects of mean TIMI frame count value and each of the MS components on HRR. A p value of $<0.05$ was regarded as statistically significant. The SPSS (SPSS Inc., Chicago, IL, USA) version 17.0 statistical package was used for all analyses.

\section{Results}

Baseline clinical characteristics of patients with MS and control patients are presented in Table 1. Patients with MS had higher systolic and diastolic $\mathrm{BP}$, BMI, waist circumference, serum TG, and fasting plasma glucose and lower HDL-C compared to control subjects, as expected. METs value $(9.4 \pm$ \pm 3.3 vs. $11.9 \pm 2.7 ; \mathrm{p}=0.02)$ was significantly lower in patients with MS, as well. However, we found no significant difference between the two groups in respect to age, the status of cigarette 
Table 1. Demographic and laboratory findings of the patients with metabolic syndrome (MS) and the controls.

\begin{tabular}{lccc}
\hline & Control group (n = 37) & MS group (n = 43) & P \\
\hline Age & $51 \pm 8$ & $55 \pm 10$ & 0.68 \\
Gender (male/female) & $22(59 \%) / 15(41 \%)$ & $22(51 \%) / 21(49 \%)$ & 0.34 \\
Smoking & $19 / 18(51 \%)$ & $16 / 27(37 \%)$ & 0.14 \\
Body mass index & $27 \pm 2$ & $30 \pm 3$ & $\mathbf{0 . 0 0}$ \\
Waist circumference [cm] & $89 \pm 8$ & $100 \pm 8$ & $\mathbf{0 . 0 0}$ \\
Systolic blood pressure [mm/Hg] & $128 \pm 25$ & $141 \pm 20$ & $\mathbf{0 . 0 1}$ \\
Diastolic blood pressure [mm/Hg] & $77 \pm 12$ & $82 \pm 13$ & $\mathbf{0 . 0 5}$ \\
Resting heart rate [bpm] & $74 \pm 13$ & $78 \pm 13$ & 0.19 \\
Peak exercise herat rate [bpm] & $158 \pm 17$ & $151 \pm 18$ & 0.10 \\
Exercise capacity [METs] & $11.9 \pm 2.7$ & $9.4 \pm 3.3$ & $\mathbf{0 . 0 2}$ \\
Fasting glucose [mg/dL] & $97 \pm 13$ & $115 \pm 29$ & $\mathbf{0 . 0 1}$ \\
Total cholesterol [mg/dL] & $204 \pm 49$ & $188 \pm 44$ & 0.13 \\
LDL cholesterol [mg/dL] & $137 \pm 42$ & $129 \pm 32$ & 0.36 \\
HDL cholesterol [mg/dL] & $44 \pm 12$ & $35 \pm 7$ & $\mathbf{0 . 0 1}$ \\
Triglyceride [mg/dL] & $113 \pm 33$ & $157 \pm 48$ & $\mathbf{0 . 0 6}$ \\
Hemoglobin [g/dL] & $14 \pm 1$ & $14 \pm 1$ & 0.71 \\
Creatinine [mg/dL] & $0.8 \pm 0.1$ & $0.8 \pm 0.1$ & 0.18 \\
Insulin resistance (HOMA-R) & $2.3 \pm 1.8$ & $3.5 \pm 2.1$ & 0.13 \\
\hline
\end{tabular}

LDL — low density lipoprotein; HDL — high density lipoprotein; METs — metabolic equivalent units

Table 2. Comparison of the heart rate recovery (HRR) and the Thrombolysis in Myocardial Infarction (TIMI) frame counts values between two groups.

\begin{tabular}{|c|c|c|c|}
\hline & $\begin{array}{c}\text { Control } \\
\text { group }(n=37)\end{array}$ & $\begin{array}{l}\text { Metabolic syndrome } \\
\text { group }(n=43)\end{array}$ & $\mathbf{P}$ \\
\hline HRR $1^{\text {st }} \min [\mathrm{bpm}]$ & $37 \pm 10$ & $32 \pm 9$ & 0.01 \\
\hline HRR $2^{\text {nd }} \min [\mathrm{bpm}]$ & $52 \pm 11$ & $46 \pm 11$ & 0.03 \\
\hline HRR $3^{\text {rd }} \min [\mathrm{bpm}]$ & $59 \pm 12$ & $51 \pm 12$ & 0.00 \\
\hline HRR $4^{\text {th }} \min [\mathrm{bpm}]$ & $61 \pm 2$ & $54 \pm 13$ & 0.02 \\
\hline Left anterior descending coronary artery & $39 \pm 15$ & $51 \pm 24$ & 0.009 \\
\hline Left circumflex coronary artery & $24 \pm 7$ & $32 \pm 11$ & 0.001 \\
\hline Right coronary artery & $24 \pm 10$ & $33 \pm 14$ & 0.003 \\
\hline Mean TIMI frame count & $29 \pm 9$ & $38 \pm 15$ & 0.002 \\
\hline
\end{tabular}

smoking, total cholesterol, hemoglobin, creatinine, and HOMA-R (Table 1).

All HRR values calculated at the first, second, third, and fourth minutes were detected significantly lower in the MS group compared to the control group $\left(H R R 1^{\text {st }}: 32 \pm 9\right.$ vs. $37 \pm 10 ; p=0.01, H R R 2^{\text {nd }}: 46 \pm 11$ vs. $52 \pm 11 ; \mathrm{p}=0.03, \mathrm{HRR} 3^{\text {rd }}: 51 \pm 12$ vs. $59 \pm 12$; $\mathrm{p}=0.00$, HRR $4^{\text {th }}: 54 \pm 13$ vs. $\left.61 \pm 2 ; \mathrm{p}=0.02\right)$ (Table 2). The interobserver variability for the TIMI frame count measurement was $8 \%$. The TIMI frame counts for each major epicardial coronary artery and mean TIMI frame count were found to be significantly higher in the MS group compared to the control group
(LAD: $51 \pm 24$ vs. $39 \pm 15 ; \mathrm{p}=0.009, \mathrm{LCx}: 32 \pm 11$ vs. $24 \pm 7 ; \mathrm{p}=0.001$, RCA: $33 \pm 14$ vs. $24 \pm 10$; $\mathrm{p}=0.003$, mean TIMI frame count: $38 \pm 15 \mathrm{vs} .29 \pm 9$; $\mathrm{p}=0.002$ ) (Table 2). In addition, the TIMI frame counts for all three major coronary vessels were found to be significantly correlated with each other. Significant negative correlations were also detected between HRR $1^{\text {st }}$ minute and coronary TIMI frame count values in patients with MS (Table 3, Fig. 1).

The results of multivariate linear regression analysis showed that none of MS parameters affected the HRR values, however mean TIMI frame count independently associated with the HRR 
Table 3. Correlations between heart rate recovery (HRR) $1^{\text {st }}$ minute and coronary Thrombolysis in Myocardial Infarction (TIMI) frame count values in patients with metabolic syndrome.

\begin{tabular}{lcc}
\hline & \multicolumn{2}{c}{ HRR $\mathbf{1}^{\text {st }}$ minute } \\
\cline { 2 - 3 } & Correlation coefficients $(\mathbf{r})$ & $\mathbf{P}$ \\
\hline Left anterior descending coronary artery & -0.33 & 0.02 \\
Left circumflex coronary artery & -0.34 & 0.02 \\
Right coronary artery & -0.38 & 0.01 \\
Mean TIMl frame count & -0.38 & 0.01 \\
\hline
\end{tabular}
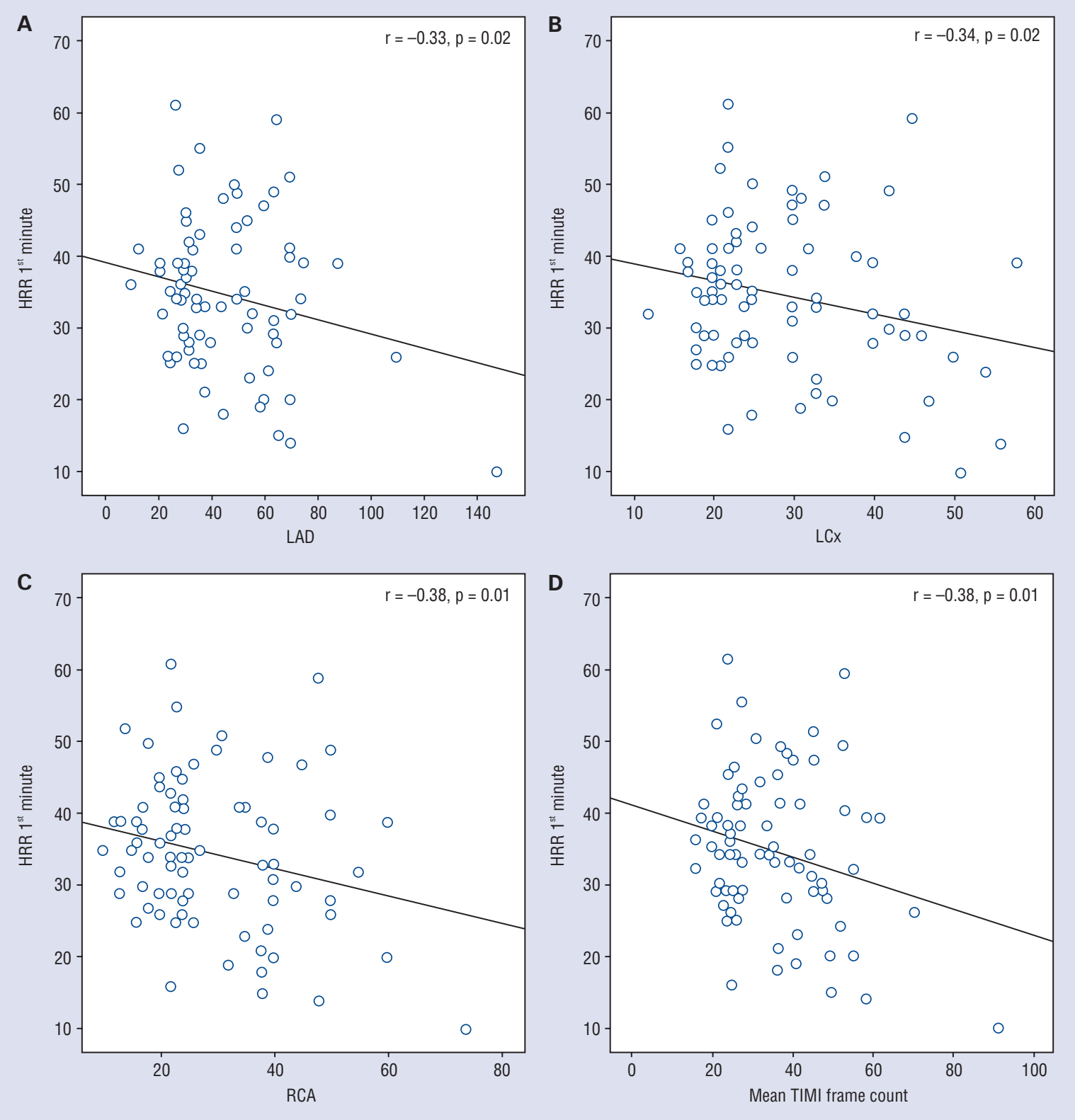

Figure 1A-D. Significant negative correlations between heart rate recovery (HRR) $1^{\text {st }}$ minute and coronary Thrombolysis in Myocardial Infarction (TIMI) frame count values in patients with metabolic syndrome (MS) (squares: MS, circles: non-MS); LAD — left anterior descending coronary artery; LCx — left circumflex coronary artery; RCA — right coronary artery. 
Table 4. Role of mean Thrombolysis in Myocardial Infarction (TIMI) frame count and each of the parameters of metabolic syndrome (MS) on heart rate recovery $1^{\text {st }}$ minute in two groups.

\begin{tabular}{lcccc}
\hline & $\begin{array}{c}\text { Control group } \\
\text { Coefficients }(\mathbf{r})\end{array}$ & $\mathbf{P}$ & $\begin{array}{c}\text { MS group } \\
\text { Coefficients }(\mathbf{r})\end{array}$ & $\mathbf{P}$ \\
\hline Mean TIMl frame count & -0.02 & 0.9 & -0.27 & $\mathbf{0 . 0 4}$ \\
Waist circumference $[\mathrm{cm}]$ & -0.14 & 0.5 & -0.33 & 0.85 \\
Systolic blood pressure $[\mathrm{mm} \mathrm{Hg]}$ & -0.17 & 0.08 & -0.10 & 0.17 \\
Diastolic blood pressure [mm Hg] & 0.36 & 0.08 & 0.18 & 0.17 \\
Fasting glucose [mg/dL] & -0.15 & 0.27 & -0.03 & 0.96 \\
High density lipoprotein cholesterol [mg/dL] & 0.25 & 0.83 & 1.7 & 0.40 \\
Triglyceride [mg/dL] & 0.02 & 0.9 & 0.39 & 0.35 \\
\hline
\end{tabular}

$1^{\text {st }}$ minute $(\mathrm{r}:-0.27, \mathrm{p}=0.04)$ in patients with MS. On the other hand, neither MS parameters nor mean frame count affected any HRR values in the control group at a statistically significant level (Table 4).

\section{Discussion}

In the literature, decreased HRR values are mostly demonstrated for the first, second, and, rarely, third minutes of the recovery period after exercise testing. In this study, we calculated HRR values at first, second, third, and fourth minutes during the recovery period after a maximal exercise test. The results obtained in this study are as follows. First, we determined that all HRR values calculated were impaired in patients with MS compared to an age and sex matched control group who did not meet the diagnostic criteria for MS. Second, the TIMI frame counts for each major epicardial coronary artery and mean TIMI frame count were found to be significantly higher in the MS group compared to the controls. Third, a significant negative correlation was also detected between mean TIMI frame count and HRR $1^{\text {st }}$ minute in patients with MS. And finally, we also found that mean TIMI frame count was the only independent predictor of decreased HRR in patients with MS. To our knowledge, this is the first study indicating this association between mean TIMI frame count and HRR in MS patients. This finding, in addition to the decreased HRR values, may especially help to identify the patients who have potentially increased CV risk despite having angiographically proven normal coronary arteries.

The association between MS and increased risk for $\mathrm{CV}$ disease has been well established in the previous studies [15-18]. It was determined that MS has added significant prognostic information to the established risk factors for CV disease, such as smoking, diabetes, hypertension, and hyperlipidemia [19]. Slow deceleration of HR after exercise was shown to indicate autonomic dysfunction [20] and was found to be an independent predictor of CV disease and all-cause mortality [3, 4]. This autonomic dysfunction, including impaired vagal reactivation, as well as sympathetic overactivity, is known to be associated with hyperinsulinemia or insulin resistance, which determines MS [5-7]. Exercise capacity and HRR were detected to be significantly impaired in young adult males with MS, by Deniz et al. [21]. It was indicated in the literature that exercise training induced favorable changes in HRR in obese men with MS [22], while reduced exercise capacity was related to cardiometabolic risk factors even in young populations [23]. Furthermore, it was shown that there was a relationship between MS and cardiac autonomic dysfunction, as detected by HR turbulence in non-diabetic patients [24]. Epicardial fat tissue is known to have unique endocrine and paracrine functions, and impairs the cardiac autonomic system, so its thickness was shown to be associated with blunted HRR in patients with MS [25]. It was also determined that decreased HRR was independently associated with MS after adjustment for the resting HR [9]. In our study as well, we detected that exercise capacity was lower in patients with MS than in the control group, however, there was no significant difference between the two groups in terms of both baseline and peak exercise HR values.

An impaired HRR at the first minute after graded exercise has been shown to be a powerful predictor of overall mortality, independent of workload, changes in HR during exercise, and the presence or absence of myocardial perfusion defects [8]. It was suggested in another study that decreased HRR occurred after, but not before, the presence of MS [26]. It was thought that decreased HRR in the presence of MS components is one 
possible mechanism by which MS is associated with increased CV disease morbidity and mortality [27]. In several studies, HRR has also been found to be associated with individual components of MS, such as blood glucose [9, 28], low HDL-C [8, 9], and resting systolic and diastolic BP [9]. In our study, we found such an association between HRR and fasting glucose and systolic BP values.

It is well known that endothelium plays an important role in the control of coronary blood flow by regulating coronary vascular resistance. Several $\mathrm{CV}$ risk factors associated with insulin resistance, comprising the MS, may be accompanied by endothelial dysfunction even in morphologically intact vessels before the onset of clinically manifested vascular disease $[10,11]$. Coronary microvasculature are the major source of coronary vascular resistance due to their small diameters and well-developed media [29]. It was stipulated in a study that the presence of increased TIMI frame counts in patients with MS with angiographically proven normal coronary arteries may be the result of impaired vessel function indicating microvascular dysfunction [12]. The effect of the autonomic nervous system on coronary blood flow and insulin resistance is well known; however, it has not been exactly understood yet whether impaired coronary blood flow occurring in MS arises from endothelial dysfunction or autonomic dysfunction.

\section{Limitations of the study}

The main limitations of this study are the small number of subjects and also its retrospective nature. The next limitation of our study is that the angiographic definition of normal coronary arteries depends on contrast angiograms of the vessel lumen, which may underestimate the presence of atherosclerotic plaque [30]. The atherosclerosis, which is associated with medial atrophy and vessel wall dilatation resulting in diffusely diseased coronary arteries, may not be visualized in normal coronary angiography [31]. Some new imaging modalities, such as intravascular ultrasound and optical coherence tomography, overcome the limitation of angiography with tomographic images that provide an accurate characterization of vessel lumen and wall geometry as well as the presence and distribution of atherosclerosis.

\section{Conclusions}

We have shown in our study that the TIMI frame counts for each major epicardial coronary artery and mean TIMI frame count indicating impaired coronary blood flow and all HRR values calculated indicating autonomic dysfunction were significantly higher in the MS group compared to the control group, a population that consists of the participants with angiographically normal coronary arteries. This angiographic finding might be considered a sign of coronary microvascular endothelial dysfunction in patients with MS. We have also detected, for the first time in the literature, that there was a significant negative correlation between mean TIMI frame count and HRR $1^{\text {st }}$ minute, and that mean TIMI frame count was the only parameter independently associated with the HRR $1^{\text {st }}$ minute in patients with MS. Therefore, impaired coronary blood flow occurring in MS might be a clue of the autonomic dysfunction in addition to endothelial dysfunction. We believe that this significant association between mean TIMI frame count and HRR, in addition to reduced HRR, may be used as an early marker for increased CV morbidity and mortality in these subjects and thus would have a high value in the subsequent guidance of the diagnosis and treatment. However, further research with a greater patient population is needed to show the reliability of this association.

\section{Conflict of interest: none declared}

\section{References}

1. Arai Y, Saul JP, Albrecht P et al. Modulation of cardiac autonomic activity during and immediately after exercise. Am J Physiology, 1989; 256: 132-141.

2. Savin WM, Davidson DM, Haskell WL. Autonomic contribution to heart rate recovery from exercise in human. J Applied Physiology, 1982; 53: 1572-1575.

3. Cole CR, Blackstone EH, Pashkow FJ, Snader CE, Lauer MS. Heart-rate recovery immediately after exercise as a predictor of mortality. N Engl J Med, 1999; 341: 1351-1357.

4. Mora S, Redberg EF, Sharrett AR, Blumenthal RS. Enhanced risk assessment in asymptopmatic individuals with exercise testing and Framingham risk scores. Circulation, 2005; 112: 1566-1572.

5. Emdin M, Gastaldelli A, Muscelli E et al. Hyperinsulinemia and autonomic nervous system dysfunction in obesity: Effects of weight loss. Circulation, 2001; 103: 513-519.

6. Esler M, Rumantir M, Wiesner G, Kaye D, Hastings J, Lambert G. Sympathetic nervous system and insulin resistance: from obesity to diabetes. Am J Hypertens, 2001; 14: 304S-309S.

7. Straznicky NE, Eikelis N, Lambert EA, Esler MD. Mediators of sympathetic activation in metabolic syndrome obesity. Curr Hypertens Rep, 2008; 10: 440-447.

8. Lind L, Andren B. Heart rate recovery after exercise is related to the insulin resistance syndrome and heart rate variability in elderly men. Am Heart J, 2002; 144: 666-672.

9. Sung J, Choi YH, Park JB. Metabolic syndrome is associated with delayed heart rate recovery after exercise. J Korean Med Sci, 2006; 21: 621-626. 
10. Harrison DG. Endothelial dysfunction in the coronary microcirculation: a new clinical entity or an experimental finding? J Clin Invest, 1993; 91: 1-2.

11. Arcaro G, Zamboni M, Rossi L et al. Body fat distribution predicts the degree of endothelial dysfunction in uncomplicated obesity. Int J Obes Relat Metab Disord, 1999; 23: 936-942.

12. Turhan H, Erbay AR, Yasar AS, Bicer A, Sasmaz H, Yetkin E. Impaired coronary blood flow in patients with metabolic syndrome: Documented by Thrombolysis in Myocardial Infarction (TIMI) frame count method. Am Heart J, 2004; 148: 789-794.

13. Gibson CM, Cannon CP, Daley WL et al. TIMI frame count: A quantitative method of assessing coronary artery flow. Circulation, 1996; 93: 879-888.

14. Matthews DR, Hosker JP, Rudenski AS, Naylor BA, Treacher DF, Turner RC. Homeostasis model assessment: insulin resistance and beta-cell function from fasting plasma glucose and insulin concentrations in man. Diabetologia, 1985; 28: 412-419.

15. Lakka HM, Laaksonen DE, Lakka TA et al. The metabolic syndrome and total and cardiovascular mortality in middle aged men. J Am Med Assoc, 2002; 288: 2709-2716.

16. Isomaa B, Almgren P, Tuomi T et al. Cardiovascular morbidity and mortality associated with the metabolic syndrome. Diabetes Care, 2001; 24: 683-689.

17. Malik S, Wong ND, Franklin SS et al. Impact of the metabolic syndrome on mortality from coronary heart disease, cardiovascular disease, and all causes in United States adults. Circulation, 2004; 110: 1245-1250.

18. Katzmarzyk PT, Church TS, Blair SN. Cardiorespiratory fitness attenuates the effects of the metabolic syndrome on all-cause and cardiovascular disease mortality in men. Arch Int Med, 2004; 164: 1092-1097.

19. Sundstrom J, Riserus U, Byberg L, Zethelius B, Lithell H, Lind L. Clinical value of the metabolic syndrome for long term prediction of total and cardiovascular mortality: Prospective, population based cohort study. Br Med J, 2006; 332: 878-882.

20. Manfrini O, Pizzi C, Trerè D, Fontana F, Bugiardini R. Parasympathetic failure and risk of subsequent coronary events in unstable angina and non-ST-segment elevation myocardial infarction. Eur Heart J, 2003; 24: 1560-1566.
21. Deniz F, Katircibasi MT, Pamukcu B, Binici S, Sanisoglu SY. Association of metabolic syndrome with impaired heart rate recovery and low exercise capacity in young male adults. Clin Endocrinol (Oxf), 2007; 66: 218-223.

22. Kim MK, Tanaka K, Kim MJ, Matsuo T, Ajisaka R. Exercise training-induced changes in heart rate recovery in obese men with metabolic syndrome. Metab Syndr Relat Disord, 2009; 7: 469-476.

23. Peterson MD, Liu D, IglayReger HB, Saltarelli WA, Visich PS, Gordon PM. Principal component analysis reveals gender-specific predictors of cardiometabolic risk in $6^{\text {th }}$ graders. Cardiovasc Diabetol, 2012; 11: 146.

24. Erdem A, Uenishi M, Küçükdurmaz $Z$ et al. The effect of metabolic syndrome on heart rate turbulence in non-diabetic patients. Cardiol J, 2012; 19: 507-512.

25. Sengul C, Duman D. The association of epicardial fat thickness with blunted heart rate recovery in patients with metabolic syndrome. Tohoku J Exp Med, 2011; 224: 257-262.

26. Kizilbash MA, Carnethon MR, Chan C, Jacobs DR, Sidney S, Liu K. The temporal relationship between heart rate recovery immediately after exercise and the metabolic syndrome: The CARDIA study. Eur Heart J, 2006; 27: 1592-1596.

27. Sattar N, Gaw A, Scherbakova O et al. Metabolic syndrome with and without C-reactive protein as a predictor of coronary heart disease and diabetes in the West of Scotland Coronary Prevention Study. Circulation, 2003; 108: 414-419.

28. Panzer C, Lauer MS, Brieke A, Blackstone E, Hoogwerf B. Association of fasting plasma glucose with heart rate recovery in healthy adults: A population-based study. Diabetes, 2002; 51: 803-807.

29. Epstein SE, Cannon O, Talbot TL. Hemodynamic principles in the control of coronary blood flow. Am J Cardiol, 1985; 56: 4E-10E.

30. Arnett EN, Isner JM, Redwood DR et al. Coronary artery narrowing in coronary heart disease: Comparison of cineangiographic and necropsy findings. Ann Intern Med, 1979; 91: 350-356.

31. Chemarin-Alibelli MJ, Pieraggi MT, Elbaz M et al. Identification of coronary thrombus after myocardial infarction by intracoronary ultrasound compared with histology of tissues sampled by atherectomy. Am J Cardiol, 1996; 77: 344-349. 